\title{
Light-induced order-of-magnitude decrease in the electric field for domain nucleation in MgO-doped lithium niobate crystals
}

\author{
C. L. Sones ${ }^{\text {a) }}$ \\ Optoelectronics Research Centre, University of Southampton, Southampton, SO17 1BJ, United Kingdom \\ M. C. Wengler \\ Institute of Physics, University of Bonn, Wegelerstrasse 8, D 53115 Bonn, Germany \\ C. E. Valdivia, S. Mailis, and R. W. Eason \\ Optoelectronics Research Centre, University of Southampton, Southampton, SO17 1BJ, United Kingdom \\ K. Buse \\ Institute of Physics, University of Bonn, Wegelerstrasse 8, D 53115 Bonn, Germany
}

(Received 13 January 2005; accepted 1 April 2005; published online 16 May 2005)

\begin{abstract}
We report an order-of-magnitude reduction in the electric field required for domain nucleation in $1 \mathrm{~mol} \% \mathrm{MgO}$-doped near-stoichiometric and $5 \mathrm{~mol} \% \mathrm{MgO}$-doped congruently grown lithium niobate crystals induced by illumination from a focused continuous wave laser beam at wavelengths of 514,488 , and $457 \mathrm{~nm}$. A smaller decrease of $31 \%$ is also observed for undoped congruently grown crystals. The effect is independent of the visible wavelengths explored. Light-controlled domain patterning is also demonstrated. (C) 2005 American Institute of Physics.
\end{abstract}

[DOI: 10.1063/1.1929099]

Periodically poled lithium niobate (PPLN) has been successfully exploited for various quasi-phase-matched nonlinear applications. ${ }^{1}$ The efficiency of the quasi-phase-matching is limited, however, by the susceptibility of congruently grown lithium niobate to photorefractive damage ${ }^{2,3}$ induced during room temperature operation with high power and short-pulsed lasers, restricting the applicability of congruently grown lithium niobate. This limitation can be significantly reduced by the use of magnesium-doped ( $\mathrm{MgO}-$ doped) lithium niobate which exhibits a higher resistance to such photorefractive effects, ${ }^{4,5}$ a lower coercive field for ferroelectric domain reversal, ${ }^{6}$ and comparable nonlinear optical coefficients ${ }^{7}$ for use in frequency conversion processes. ${ }^{8}$ Electric field poling with structured electrodes is a routinely employed technique for fabrication of PPLN, but the period lengths obtainable with this technique are currently limited to a few micrometers. However, many applications require smaller structures, hence alternative methods for domain patterning are currently under investigation in our laboratory.

Light-induced domain patterning may be a suitable technique to overcome this size limitation. Previous lightassisted poling experiments which take advantage of an ultraviolet-light-induced change in the coercive field to transfer a patterned light distribution into an equivalent domain structure in bulk crystals have already been reported for undoped and $\mathrm{MgO}$-doped lithium niobate ${ }^{9-11}$ crystals. The use of focused visible laser light, which has the effect of reducing the coercive field through a light-induced space charge field, has recently been demonstrated to directly write domain structures of $\sim 2 \mu \mathrm{m}$ dimensions in undoped lithium niobate crystals. ${ }^{12}$

Here we investigate the influence of the illumination by visible light from an Ar-ion laser on the electric field required to induce domain nucleation (nucleation field) in $\mathrm{MgO}-$ doped and undoped lithium niobate crystals. Domain

\footnotetext{
a) Author to whom correspondence should be addressed; electronic mail: cls@orc.soton.ac.uk
}

nucleation is defined here as the first observation of $180^{\circ}$ inverted domain seeds that subsequently extend through the crystal on further application of an electric field. It is worth noting that the nucleation field is less than the coercive field, which is often defined by a poling current exceeding some arbitrary threshold, as in Ref. 11.

Several types of commercially available $z$-cut single crystal lithium niobate samples from different crystal suppliers were investigated. The two types of congruently grown crystals used were $300-\mu \mathrm{m}$-thick undoped (YamPure) and 500 - $\mu \mathrm{m}$-thick $5 \mathrm{~mol} \% \mathrm{MgO}$-doped (YamMg50) lithium niobate crystals from Yamaju Ceramics, Japan. The nearstoichiometric crystals used were 500- $\mu \mathrm{m}$-thick $1 \mathrm{~mol} \%$ $\mathrm{MgO}$-doped (DelMg10) lithium niobate crystals from Deltronic, USA. The observed shift of the $\mathrm{OH}$ peak in the measured absorption spectra for the $\mathrm{MgO}$-doped samples (peak at $2830 \mathrm{~nm}$ for YamMg50 and DelMg10, compared to 2870 $\mathrm{nm}$ for YamPure) indicate that their dopant concentrations were above the so-called optical damage threshold. ${ }^{13}$

To study the influence of light on the nucleation of domains and their subsequent domain inversion during the electric field poling, a specially designed fused silica sample holder was used. ${ }^{9,14}$ This cell allowed the use of transparent liquid (water) electrodes required for the simultaneous illumination and application of a uniform electric field along the $z$ axis of the crystal. The experimental setup, shown in Fig. 1, involved a lens with a focal length of $10 \mathrm{~cm}$ that focused a spatially filtered and collimated beam from an Ar-ion laser on the $-z$ face of the $z$-cut lithium niobate crystals. The measured spot radius of the focused laser beam was $\sim 45 \mu \mathrm{m}$. This choice of illuminating laser spot size was a compromise between sufficiently high intensity and large spatial extent. As the Rayleigh range for the focused beam $(\sim 3 \mathrm{~mm})$ was much larger than the crystal thickness, precise positioning of the crystal at the focal point of the lens was not critical for our experimental study, and also ensured a relatively uniform spatial distribution over the entire crystal thickness. The Arion laser wavelengths used were $\lambda=514,488$, and $457 \mathrm{~nm}$, 


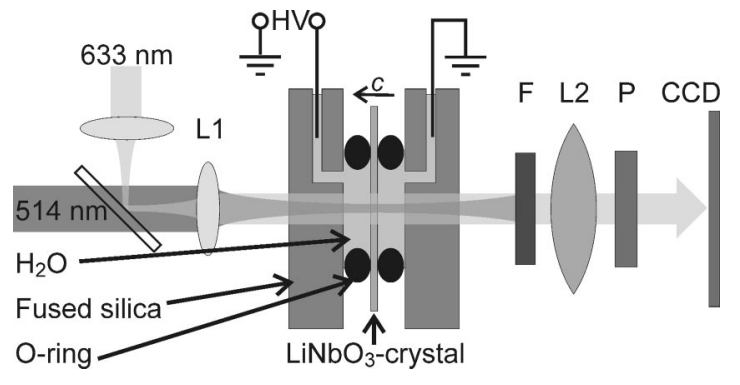

FIG. 1. Schematic of the experimental setup used for light-assisted electricfield poling experiments. (HV: high voltage supply, L1: collimating and focusing lens, F: red filter, L2: imaging lens, P: polarizer).

for which the lithium niobate crystals were measured to have low absorption $\left(\alpha<0.005 \mathrm{~mm}^{-1}\right)$. The incident power was varied up to $200 \mathrm{~mW}$ yielding a maximum intensity of $I \approx 3 \mathrm{~kW} \mathrm{~cm}^{-2}$.

To investigate domain nucleation, a simple in situ optical imaging setup was employed using a collimated polarized beam from a HeNe laser at $\lambda=633 \mathrm{~nm}$, a crossed polarizer, and a CCD camera. The beam from the HeNe laser collinearly traversed the optical path of the illuminating beam from the Ar-ion laser. Real-time observation of the nucleation and growth of domains was possible due to the straininduced birefringence in the areas surrounding the $180^{\circ}$ domain walls. ${ }^{15}$ The applied electric field was ramped at a constant rate of $10 \mathrm{~V} \mathrm{~mm}^{-1} \mathrm{~s}^{-1}$.

The nucleation field for the first poling of a virgin crystal has a larger value than for subsequent poling. Therefore, the nucleation field in the absence of light, $E^{\text {nucl }}(I=0)$, was determined by successively poling the crystals a minimum of five times in both the forward and reverse directions after the first poling cycle, obtaining a reproducible value that was stable for at least 50 subsequent poling cycles. The crystal was then illuminated by Ar-ion laser light at different intensities, I. The nucleation field under laser illumination, $E^{\text {nucl }}(I)$, was then measured as a function of the incident laser intensity. The recorded electric field value corresponded to the first observation of a domain seed within the illuminated region.

The plots in Fig. 2 show the variation of $E^{\text {nucl }}(I)$, over a range of incident laser intensities at $514 \mathrm{~nm}$ for all three types of crystals. It is evident from these plots that the mag-

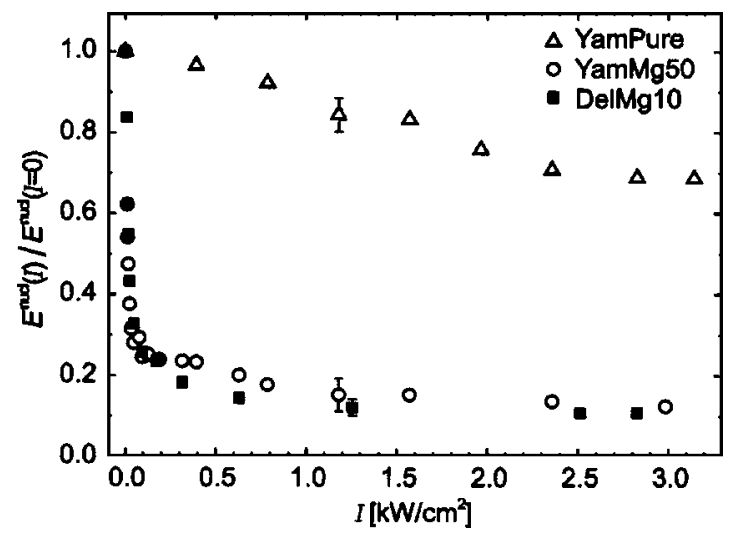

FIG. 2. Plot showing values for the electric field required for nucleation in the presence of light, $E^{\text {nucl }}(I)$, normalized relative to corresponding values required for nucleation in the absence of light, $E^{\text {nucl }}(I=0)$, with varying laser

intensities at $\lambda=514 \mathrm{~nm}$.
Downloaded 10 Nov 2009 to 152.78.208.72. Redistribution subject to AIP license or copyright; see http://apl.aip beam from an objective lens.
TABLE I. Electric field required for domain nucleation in different crystals, in the presence and absence of light at $\lambda=514 \mathrm{~nm}$.

\begin{tabular}{|c|c|c|c|}
\hline $\begin{array}{l}\text { Crystal } \\
\text { type }\end{array}$ & $\begin{array}{c}E^{\mathrm{nucl}}\left(I^{\max }\right) \text { for } \\
I^{\max } \approx 3 \mathrm{~kW} \mathrm{~cm}^{-2} \text { at } \\
\lambda=514 \mathrm{~nm}\left(\mathrm{kV} \mathrm{mm}^{-1}\right)\end{array}$ & $\begin{array}{l}E^{\text {nucl }}(I=0) \\
\left(\mathrm{kV} \mathrm{mm}^{-1}\right)\end{array}$ & Reduction \\
\hline DelMg10 & 0.28 & 2.63 & $89 \%$ \\
\hline YamMg50 & 0.78 & 6.28 & $88 \%$ \\
\hline YamPure & 14.3 & 20.9 & $31 \%$ \\
\hline
\end{tabular}

nitude of the field required for nucleation in each of the different crystals shows a systematic decrease with increasing laser intensity, and reaches an effective saturation at higher intensities.

Table I shows the values of the nucleation fields in the absence of light, $E^{\text {nucl }}(I=0)$, and for the highest available powers at $\lambda=514 \mathrm{~nm}, E^{\text {nucl}}\left(I^{\max }\right)$, in each crystal. The values indicate a light-induced one order-of-magnitude reduction of the nucleation field in the MgO-doped crystals, and a 31\% reduction for the undoped congruently grown samples. As this effect showed little, if any, dependence on the wavelengths explored $(\lambda=514,488$, and $457 \mathrm{~nm})$ in any of the crystal types examined we only present results for one of these wavelengths $(514 \mathrm{~nm})$.

The above-noted reduction was measured in the forward poling direction, however an effect of a similar magnitude was also observed for reverse poling. The effect was also found to be independent of the crystal face $(+z$ or $-z)$ chosen for illumination. The simultaneous presence of light during the application of electric field is essential for the nucleation field reduction, as no reduction was observed for poling even a few seconds after illumination.

Light-induced domain patterning was demonstrated in a YamMg50 sample. The laser beam $(\lambda=514 \mathrm{~nm})$ was focused into the crystal at an intensity of $I=1.2 \mathrm{~kW} \mathrm{~cm}^{-2}$, and a constant electric field of $E=4.0 \mathrm{kV} \mathrm{mm}^{-1}$ was applied. Under these conditions domain nucleation occurred inside the beam spot only, appearing within $\sim 1 \mathrm{~s}$. After nucleation was observed within the beam spot, the translation stage was moved manually by approximately $100 \mu \mathrm{m}$ along the $y$ axis of the crystal. This procedure was repeated to obtain a line of nucleation seeds of several millimeters in length. Figure 3(a) shows a scanning electron microscope (SEM) image of the nucleation line revealed by wet etching in hydrofluoric acid. The dashed lines represent the position of the $1 / e^{2}$ intensity of the laser spot (diameter $90 \mu \mathrm{m}$ ) during motion. These domains were observed on both faces of the crystal following etching, indicating that they were bulk domains extending through the entire thickness of the crystal, with lateral dimensions varying from 20 to $120 \mu \mathrm{m}$. Domain nucleation

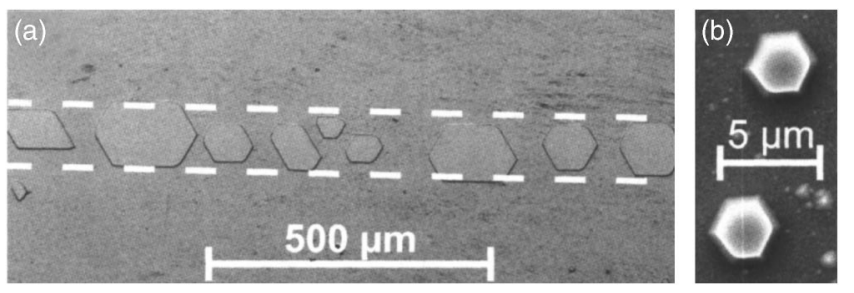

FIG. 3. SEM images showing (a) a row of bulk domains formed by repositioning the laser beam on the crystal, and (b) surface domains induced by a tightly focused beam from an objective lens. 
was always observed in arbitrary positions within the extent of the laser spot.

To investigate the possibility of inducing domain sizes of the order of a few micrometers, we further experimented with tighter focusing via a $6.3 \times$ microscope objective lens, with a spot radius of $20 \mu \mathrm{m}$. Our initial results [Fig. 3(b)] show surface domains, as revealed by differential etching, present only on the $-z$ face, of size $\sim 2 \mu \mathrm{m}$. However use of such a short working distance objective lens meant simultaneous visualization of domain nucleation and hence further control of their growth was not possible. The small domains seen within the illuminated spot have therefore likely grown from their initial sizes at the instant of nucleation. An improved setup involving in situ monitoring should allow for the formation of even smaller domains.

The origin of the observed light-induced reduction of the electric field required for domain nucleation has not yet been clarified. In MgO-doped crystals, the measured reduction of the nucleation field is significantly greater than the corresponding coercive field reduction of $34 \%$ reported using ultraviolet wavelengths. ${ }^{11}$ However, a hysteresis loop in Ref. 10 also shows a small poling current for as little as $E_{\text {applied }}$ $=3 \mathrm{kV} \mathrm{mm}^{-1}$, indicating possible domain nucleation at $\sim 50 \%$ of the assumed coercive field. In undoped crystals, a visible-light-induced reduction of the coercive field has previously been reported, and has been attributed to the formation of a space charge field due to inhomogeneous illumination along the $z$ axis. ${ }^{12}$ In our case a reduction of the same magnitude $(30 \%)$ has been observed with homogeneous illumination and may be associated with a bulk photovoltaic effect. However, for undoped crystals it is unlikely that the small photovoltaic fields produced would influence the coercive field to the extent observed. Furthermore, a spacecharge field-driven mechanism cannot explain the observed three times greater influence of the light in $\mathrm{MgO}$-doped lithium niobate. ${ }^{4}$

Ishizuki et al. have demonstrated a reduction of the coercive field by $75 \%$ at elevated temperatures as high as $250{ }^{\circ} \mathrm{C}$ in $\mathrm{MgO}$-doped lithium niobate. ${ }^{16}$ Absorption coefficients of the lithium niobate crystals at the wavelengths used in our experiments have been measured to be below $0.005 \mathrm{~mm}^{-1}$ which is unlikely to lead to such a large temperature increase. Micro-Raman spectroscopy performed by Dierolf et al. ${ }^{12}$ did not reveal any temperature increase generated by light intensities similar to those used in our experiment, so any temperature mechanism can be effectively neglected.

The most significant impact of light on the nucleation field of lithium niobate was observed in $\mathrm{MgO}$-doped material. This behavior is in agreement with other studies on the ultraviolet-light-induced coercive field reduction of lithium niobate crystals. ${ }^{11}$ Pinning of domain walls has been shown to be responsible for the large coercive field in congruently grown crystals, anchoring the domain wall and preventing growth at electric fields below the observed coercive field. However, between these pinning points, domain growth and domain wall bending has been observed at electric fields an order of magnitude lower than the coercive field. ${ }^{17}$ We believe that visible light may cause reduction of the pinning strength, leading to a lower electric field required for domain nucleation. The pinning centers are proposed to be related to the crystal defects (lithium vacancies and niobium antisites). ${ }^{18}$ This could explain the large changes in the lightinduced poling behavior for $\mathrm{MgO}$-doped crystals, since this dopant modifies the defect structure of lithium niobate, ${ }^{13}$ and therefore may influence the sensitivity to the light-induced recharging of the pinning centers.

We have demonstrated light-induced domain patterning of bulk domains with a focused visible light beam. A limitation in our setup was the manual control of the laser-spot position, which only permitted the generation of nucleation at separated positions and not continuous writing of a domain line. It also led to uncontrolled domain growth when the laser spot had not been moved fast enough to a different position. The bulk domain size, however, appears to be limited mainly by the laser spot size, and surface domains of micrometer dimensions have been produced. We are confident of obtaining better control of the small domain structures by reducing the spot size and using an automated translation stage. The disadvantages of serial domain writing with a focused spot may be overcome using an interference pattern obtained by a phase mask. By this technique, we hope to produce domain structures on a micrometer periodic scale.

In conclusion, we have reported a one order-ofmagnitude reduction in the electric field required to nucleate domains in $\mathrm{MgO}$-doped crystals by visible laser light illumination, and used this effect to optically control domain nucleation. Bulk domains with sizes of several micrometers and surface domains on the micrometer scale have been produced. We believe that light-assisted electric field poling could be an ideal route to achieve controlled micrometerscale domain structuring of optical-damage resistant $\mathrm{MgO}$ doped crystals.

We are pleased to acknowledge research funding via the Engineering and Physical Sciences Research Council, UK, via Grant No. GR/S47373, and gratefully acknowledge financial support by the Deutsche Forschungsgemeinschaft, the Deutsche Telekom AG, and the Deutsche Telekom Stiftung.

${ }^{1}$ L. Arizmendi, Phys. Status Solidi A 201, 253 (2004).

${ }^{2}$ A. Ashkin, G. D. Boyd, J. M. Dziedzic, R. G. Smith, A. A. Ballman, J. J. Levinstein, and K. Nassau, Appl. Phys. Lett. 9, 72 (1966).

${ }^{3}$ K. Buse, Appl. Phys. B 64, 273 (1997).

${ }^{4}$ D. A. Bryan, R. Gerson, and H. E. Tomaschke, Appl. Phys. Lett. 44, 847 (1984).

${ }^{5}$ Y. Furukawa, K. Kitamura, S. Takekawa, K. Niwa, and H. Hatano, Opt. Lett. 23, 1892 (1998).

${ }^{6}$ A. Kuroda, S. Kurimura, and Y. Uesu, Appl. Phys. Lett. 69, 1565 (1996).

${ }^{7}$ I. Shoji, T. Kondo, A. Kitamoto, M. Shirane, and R. Ito, J. Opt. Soc. Am. B 14, 2268 (1997).

${ }^{8}$ M. Nakamura, M. Sugihara. M. Kotoh, H. Taniguchi, and K. Tadatomo, Jpn. J. Appl. Phys., Part 2 38, L1234 (1999).

${ }^{9}$ M. Müller, E. Soergel, and K. Buse, Appl. Phys. Lett. 83, 1824 (2003).

${ }^{10}$ A. Fujimura, T. Sohmura, and T. Suhara, Electron. Lett. 39, 719 (2003).

${ }^{11}$ M. C. Wengler, B. Fassbender, E. Soergel, and K. Buse, J. Appl. Phys. 96, 2816 (2004).

${ }^{12}$ V. Dierolf and C. Sandmann, Appl. Phys. Lett. 84, 3987 (2004).

${ }^{13}$ O. F. Schirmer, O. Thiemann, and M. Wohlecke, J. Phys. Chem. Solids 52, 185 (1991).

${ }^{14}$ M. C. Wengler, M. Müller, E. Soergel, and K. Buse, Appl. Phys. B 76, 393 (2003).

${ }^{15}$ V. Gopalan and T. E. Mitchell, J. Appl. Phys. 83, 941 (1998).

${ }^{16}$ H. Ishizuki, I. Shoji, and T. Taira, Appl. Phys. Lett. 82, 4062 (2003).

${ }^{17}$ T. J. Yang, V. Gopalan, P. J. Swart, and U. Mohideen, Phys. Rev. Lett. 82, 4106 (1999).

${ }^{18}$ S. Kim, V. Gopalan, K. Kitamura, and Y. Furukawa, J. Appl. Phys. 90, 2949 (2001) 